\title{
APPARENT GLAUCOMATOUS VISUAL FIELD DEFECTS CAUSED BY DERMATOCHALASIS
}

\author{
ALAN S. KOSMIN, PETER K. WISHART and MICHAEL K. BIRCH \\ Liverpool
}

\begin{abstract}
SUMMARY
We have studied the effects of dermatochalasis on Humphrey automated perimetry of the central $24^{\circ}$ visual field. Fifteen visual fields of 9 ocular hypertensive patients (18 eyes) were found to be incongruous with their apparently healthy optic discs. Examination revealed dermatochalasis, which was felt to be responsible for the field defects. This was confirmed by reversal of the defects on repeating the field test (programme 24-2) with the redundant upper lid skin taped up, or in 2 cases following blepharoplasty. The defects always involved the superior visual field. The deepest and largest defects were sited in the superotemporal quadrant in 13 of the 15 affected fields and the supero-nasal quadrant in 2 fields. The most common pattern was a temporally skewed defect which reflected the tendency of the loose upper lid skin to be greater in extent temporally than nasally. In 7 fields the superotemporal defect extended to fuse with the blind spot, mimicking a superior arcuate scotoma. Temporal extension of the field defects below the horizontal meridian occurred in 5 fields. In cases where visual field testing was repeated without taping up the lid inter-test fluctuation in scotoma size and depth was observed, although the position of scotomas when present within the visual field remained constant. We conclude that dermatochalasis has the potential to confound diagnostic automated visual field testing for glaucoma.
\end{abstract}

In the presence of a normal optic nerve head and normal visual field an eye with raised intraocular pressure is regarded as suffering from ocular hypertension. The earliest sign of glaucoma developing may be the demonstration of visual field loss, although damage to the optic disc may be detected by an experienced observer performing biomicroscopy ${ }^{1}$ prior to the development of visual

From: Glaucoma Clinic, St Paul's Eye Unit, Royal Liverpool University Hospital, Liverpool, UK.

Correspondence to: Mr Peter Wishart, St Paul's Eye Unit $(8 z$ link), Royal Liverpool University Hospital, Prescot Street, Liverpool L7 8XP, UK. Tel: +44 (0151) 706 3968. Fax: +44 (0151) 7065861 . field loss. Routine examination of both the visual fields and optic discs is the accepted way of monitoring ocular hypertensive patients so that early treatment may be given if a patient converts from ocular hypertension to glaucoma. In this study we have used automated static perimetry, as it has been shown that it can detect glaucomatous visual field loss before manual perimetry. ${ }^{2}$

Whenever visual field loss is demonstrated and examination shows the optic disc to be unchanged the clinician must consider the possibility of a nonglaucomatous cause of visual field loss being present. Many factors may affect the visual fields, such as ocular and neurological disease or artefactual loss due to miosis, ${ }^{3}$ prominent nose, ${ }^{4}$ prominent brow ${ }^{4}$ or ptosis. $^{5-8}$

A common upper lid disorder in the elderly population with potential to restrict the visual field is dermatochalasis. Dermatochalasis is a redundancy of upper lid skin which is often associated with protrusion of orbital fat through a weakened orbital septum and usually develops secondary to agerelated involutional changes. ${ }^{9}$ While superior visual field loss is a common functional indication for blepharoplasty, ${ }^{10,11}$ the effect of dermatochalasis on the central field in the context of diagnostic automated perimetry for glaucoma has not been formally studied. We report the presence of dermatochalasis of the eyelids in ocular hypertensive patients undergoing automated perimetry as a factor that may mimic glaucomatous patterns of visual field loss and confound diagnostic perimetry.

\section{PATIENTS AND METHODS}

Nine patients with bilateral ocular hypertension and spurious field loss were identified in the Glaucoma Clinic at the Royal Liverpool University Hospital.

All showed good reliability indices when tested with Humphrey automated perimetry (programme 24-2). Seven patients were male and 2 female with a mean age of 61 years (range 52-77 years). All had corrected visual acuity of $6 / 9$ or better in both eyes. 
Table I. Improvement in mean defect and corrected pattern standard deviation for the worse-affected field in each patient following the taping up of the dermatochalasis

\begin{tabular}{lccccc}
\hline & \multicolumn{2}{c}{ Initial field } & & \multicolumn{2}{c}{ Lid taped up } \\
\cline { 2 - 3 } \cline { 5 - 6 } Patient no. & MD $(\mathrm{dB})$ & CPSD $(\mathrm{dB})$ & & MD $(\mathrm{dB})$ & CPSD $(\mathrm{dB})$ \\
\hline 1 & -4.10 & 2.38 & & -2.58 & 1.5 \\
2 & -4.88 & 2.81 & & -4.10 & 1.39 \\
3 & -10.05 & 7.94 & & -1.34 & 1.6 \\
4 & -4.94 & 8.09 & & -2.18 & 1.68 \\
5 & -4.50 & 5.63 & & -2.27 & 0.00 \\
6 & -8.14 & 10.36 & & -1.14 & 0.00 \\
7 & -10.65 & 12.01 & & -3.72 & 3.43 \\
8 & -7.83 & 6.61 & & -3.69 & 1.19 \\
9 & -4.92 & 3.59 & & -1.18 & 1.26 \\
\hline
\end{tabular}

$\mathrm{MD}$, mean defect; CPSD, corrected pattern standard deviation; $\mathrm{dB}$, decibels.

No patients were on miotic pressure-lowering medication and all had pupils with diameters of at least $2.5 \mathrm{~mm}$. Seven patients were asymptomatic and 2 reported subjective intermittent visual field loss. All patients had apparently healthy discs on slit lamp biomicroscopy (+78 D lens) following mydriasis.

Lid examination revealed bilateral dermatochalasis in all cases. The inferior margin of the fold of excess skin, whilst frequently resting on the lashes in the $90^{\circ}$ meridian, did not overhang the lid margin in this meridian and temporally the overhang was no greater than $1 \mathrm{~mm}$. No patient had a significant ptosis or inverse ptosis at the time of physical examination before field testing, all patients having an upper lid margin position such that the corneal reflex to upper lid margin distance was $>2.5 \mathrm{~mm}$. All eyes had normal levator function and no gross stigmata of levator disinsertion. No other ocular or neurological abnormalities were apparent. Abnormal fields were repeated with the redundant upper lid skin taped up
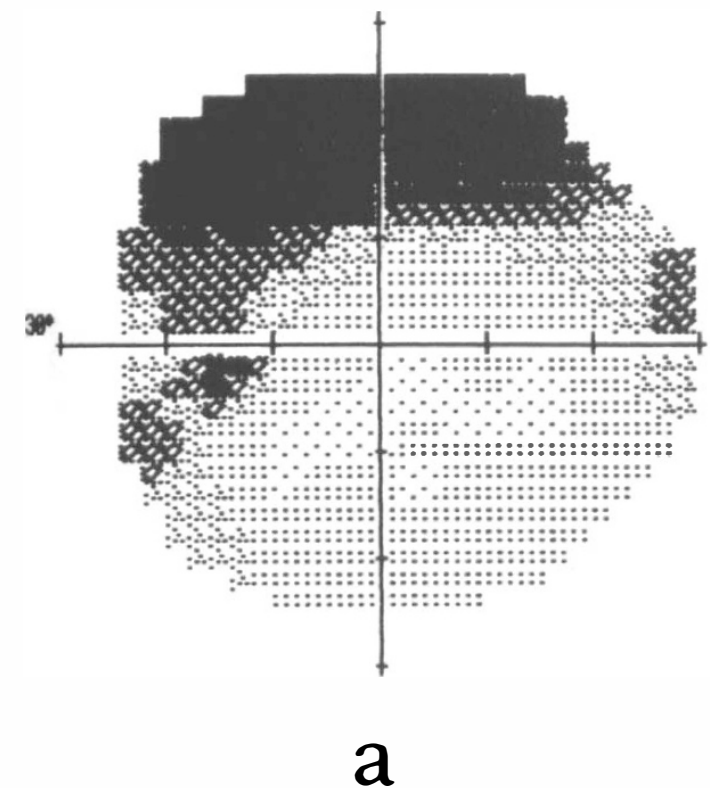

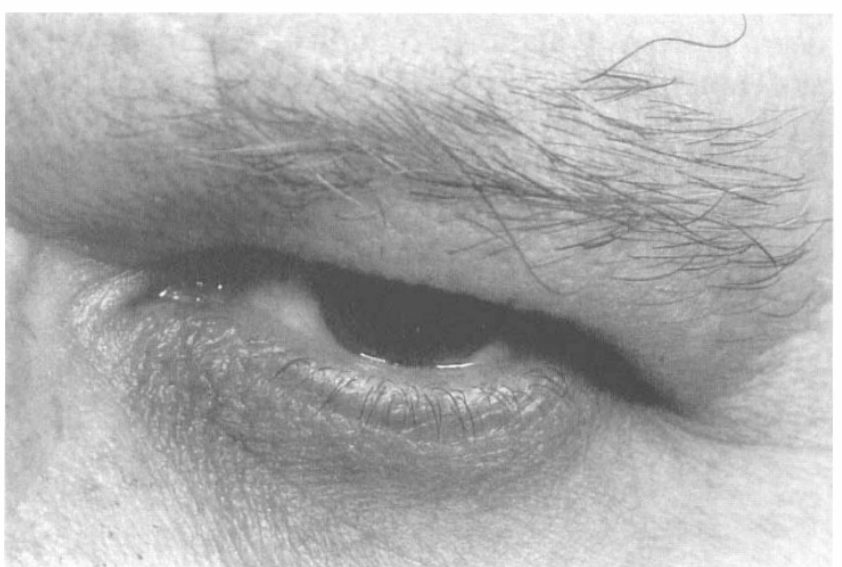

Fig. 1. Example of dermatochalasis of the left upper lid (patient 4) which was typically greater in extent on the temporal side.

and the fields compared. Patients 3, 4, 5, 6, 7 and 9 have been followed for 1-3 years. During this time their field tests were repeated without taping up the excess upper lid skin from one to four times to assess whether dermatochalasis had a consistent effect on the visual fields.

\section{RESULTS}

Abnormalities were identified in 15 of the 18 visual fields of the 9 patients with ocular hypertension. Retest of the defective visual fields with the excess upper lid skin taped up showed complete or partial reversal of the field loss in all cases. Table I shows the improvement in field mean defect (MD) and corrected pattern standard deviation (CPSD) for the more severely affected field of each patient. These figures show an improvement in average field MD from $-6.66 \mathrm{~dB}$ to $-2.46 \mathrm{~dB}$ and in CPSD from

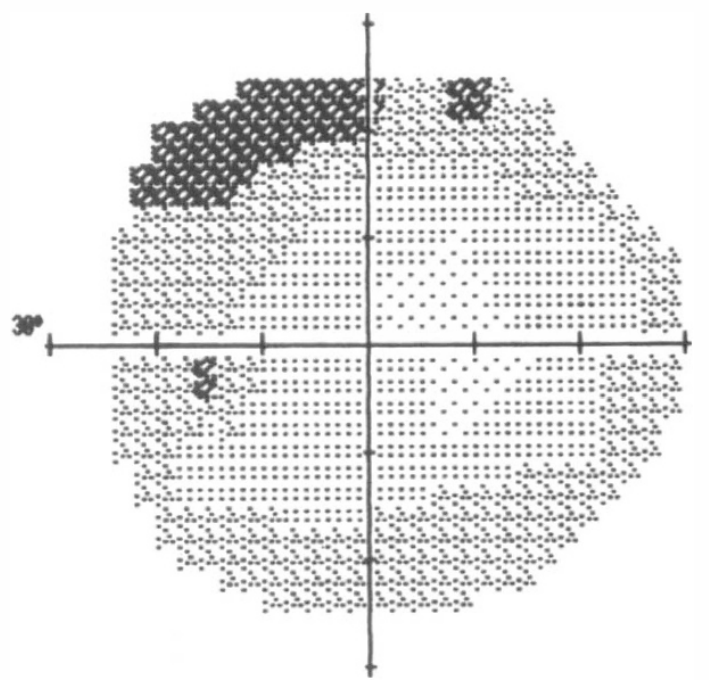

b

Fig. 2. (a) Left visual field defect associated with the dermatochalasis shown in Fig. 1. Note the temporally skewed defect typically caused by dermatochalasis. (b) Same field following the taping up of the left upper lid showing marked reversal of the defect. 


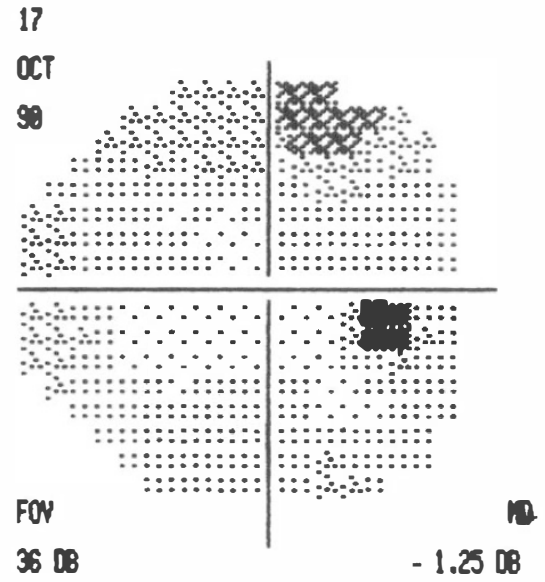

a

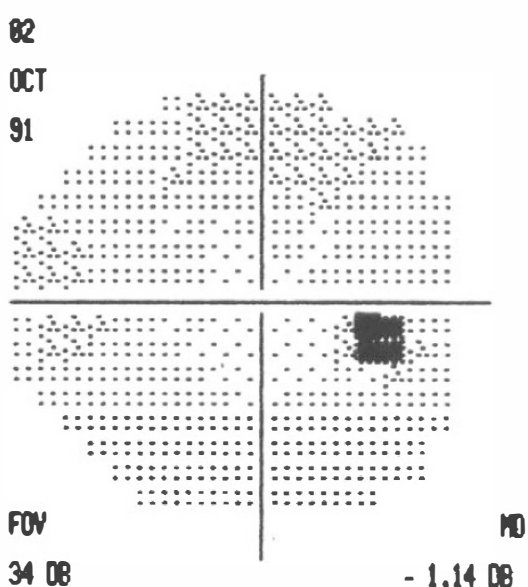

C

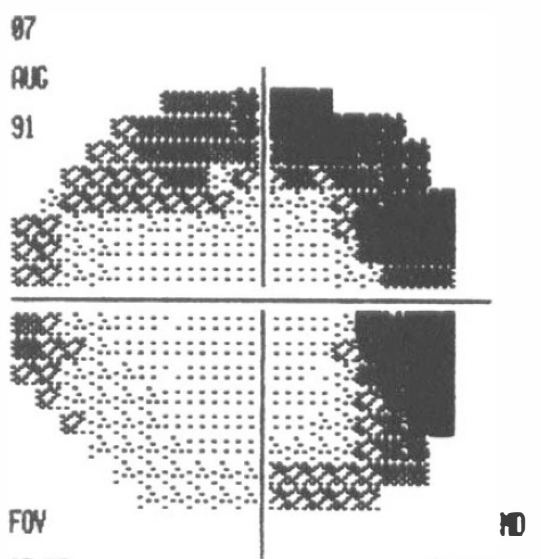

3508

$-6.9708 P<0.5$

b

68

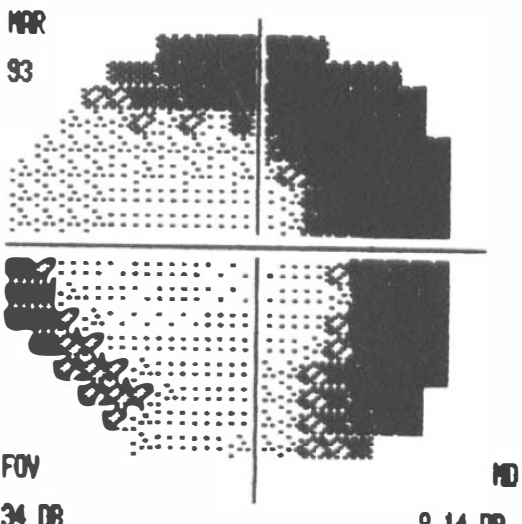

34 DB

$-8.14 \mathrm{DB} P<0.5 \%$

d

Fig. 3. (a)-(d) Series of four successive fields over 2.5 years showing intermittent right field defect associated with dermatochalasis pictured in Figs. 4 and 5.

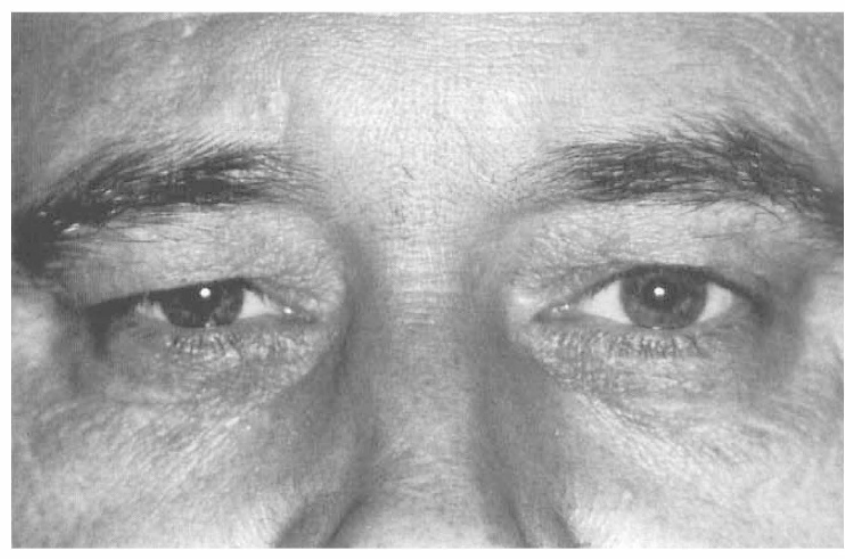

Fig. 4. En face view demonstrating bilateral dermatochalasis (right worse than left) responsible for intermittent right visual field defects shown in Fig. 3 (patient 6).

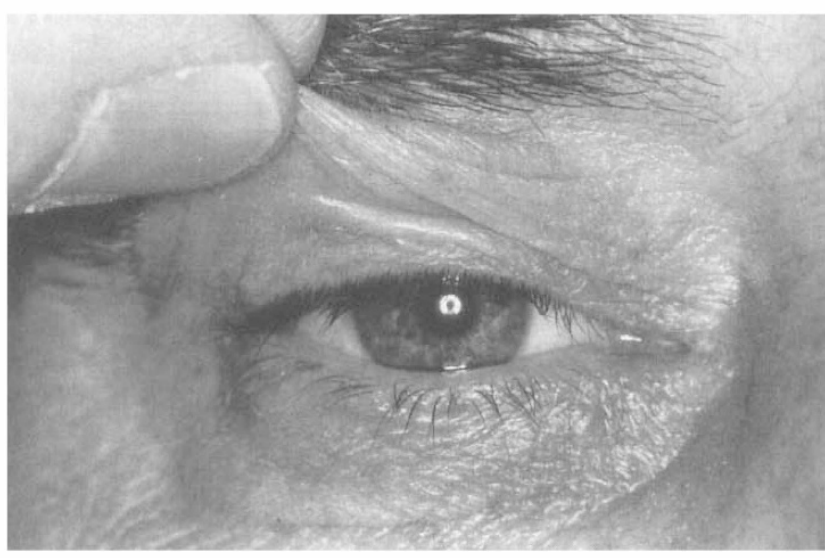

Fig. 5. Close-up view of right upper lid from Fig. 4 showing excess skin. 


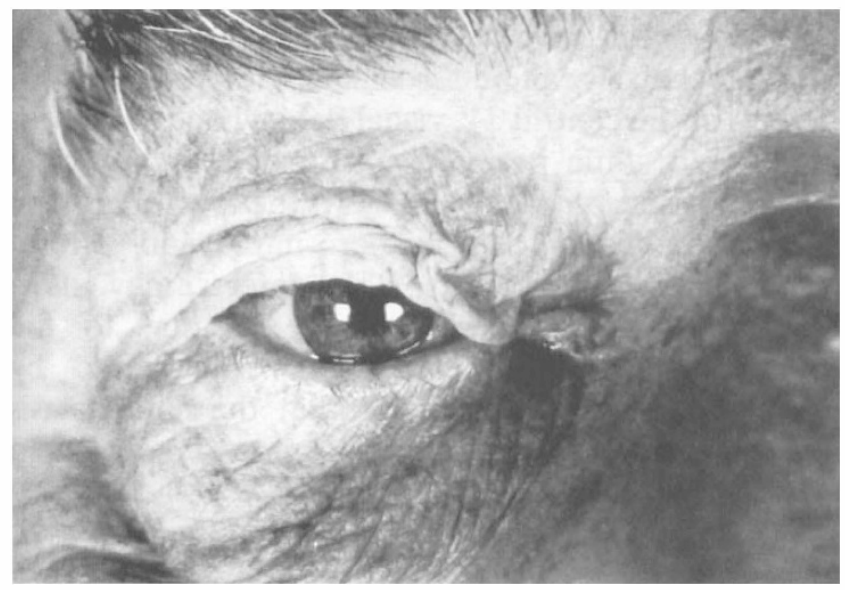

Fig. 6. Right eye of patient 8 showing dermatochalasis which atypically affected both the nasal and temporal aspects of both upper lids.

$6.60 \mathrm{~dB}$ to $1.33 \mathrm{~dB}$ when the excess upper lid skin was taped up.

The defects caused by dermatochalasis always involved the superior field, affecting both the supero-temporal and supero-nasal quadrants in all cases. The deepest and largest scotomas were located in the supero-temporal quadrant in 13 of the 15 defective fields and in the supero-nasal quadrant in 2 fields. In 7 fields the supero-temporal field defect extended to fuse with the blind spot. Extension of defects in the temporal quadrant below the horizontal meridian occurred in 5 of the 15 initial defective fields. Fig. 2 illustrates the typical field loss caused by the dermatochalasis shown in Fig. 1.
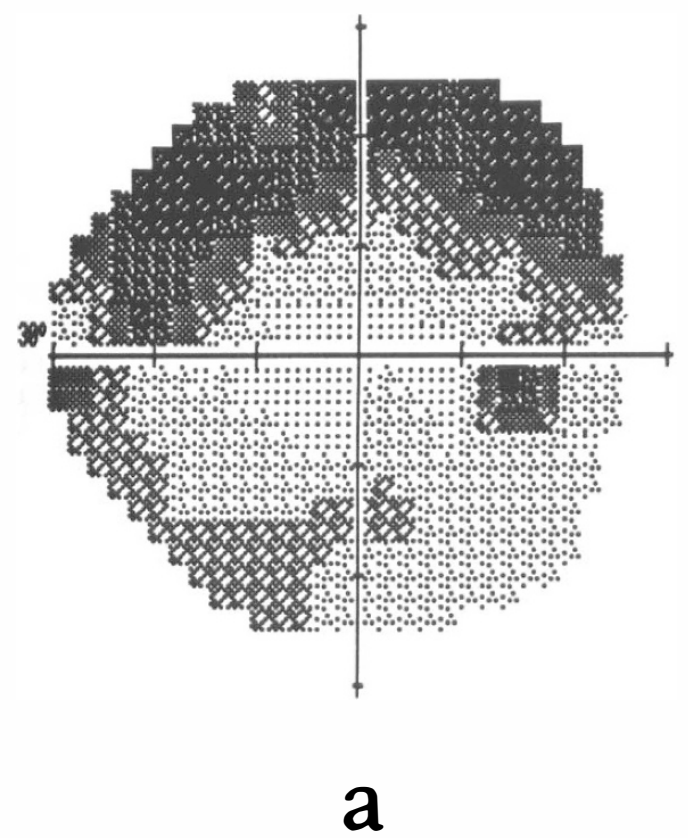

Two patients (Table I, patients 6 and 7) complained of intermittent superior visual field loss which they found to be worst when they felt fatigued. In both these cases the subjective and objective field changes disappeared following blepharoplasty procedures. Spontaneous fluctuation in the presence, size or depth of the field defect was not limited to these cases alone (Fig. 3). In fact fluctuation in performance of automated perimetry was seen in all cases where the fields were repeated on more than one clinic visit (patients 3, 4, 5, 6, 7 and 9).

\section{DISCUSSION}

While marked dermatochalasis has been well recognised as potentially interfering with the superior visual field, the 9 patients we report all had relatively mild dermatochalasis which initially could easily have gone unnoticed in the context of a glaucoma screening clinic. Despite this, the effect of dermatochalasis on the central $24^{\circ}$ visual field as tested by static Humphrey automated perimetry was significant and field defects that could be confused with glaucomatous field defects were produced.

Ocular and neurological causes of the field defects were excluded through the history and examination and we are certain that factors other than dermatochalasis known to cause artefactual field loss (e.g. miosis $^{3}$ and prominent nose ${ }^{4}$ or brow $^{4}$ ) were not responsible for the field defects, since the field loss was reversed in all cases by taping up the excess lid skin while leaving all other variables unchanged.

The field defects predominantly involved the superior visual field and were deepest in the

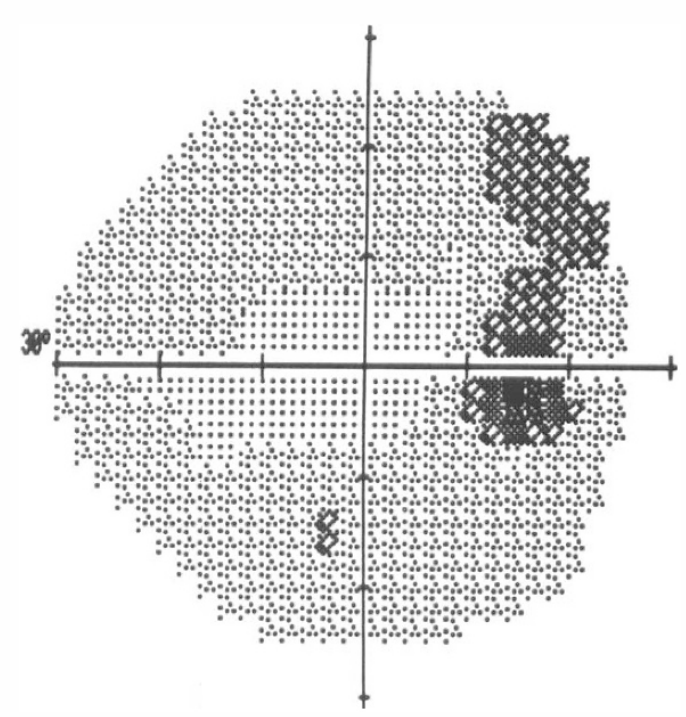

b

Fig. 7. (a) Visual field recorded from the right eye pictured in Fig. 6. A defect resembling an arcuate scotoma is shown. (b) Visual field recorded from the same eye with excess skin taped up shows almost complete disappearance of the defect. 
supero-temporal quadrant in 13 of 15 cases. The most common pattern was a temporally skewed defect (Figs. 2, 3), which reflects the tendency of the loose upper lid skin to be greater in extent temporally rather than nasally (Figs. 1, 4 and 5). We also found that even relatively mild dermatochalasis resulted in field defects which extended below the horizontal meridian in 5 of the 18 defective fields. Dermatochalasis may therefore cause scotomas in three quadrants of the visual field (Fig. 3).

It is hypothesised that in dermatochalasis the anteriorly positioned fold of skin causes field defects by blocking the passage of light into the eye. ${ }^{11}$ In addition, if the fold of excess upper lid skin were to rest down on the lashes pushing them inferiorly, the deviated lashes could either block the passage of light into the eye or cause diffraction of light from the test stimulus. This would reduce the intensity of light reaching the retina thereby making it more difficult for the subject to detect the test stimulus.

The above mechanisms for the production of field loss could be further exacerbated by coexistent mechanical $^{9}$ or aponeurotic ptosis ${ }^{11.12}$ or even pseudoptosis ${ }^{13}$ if the skin fold overhangs the eyelid margin. Intermittent expression of a mechanical ptosis could exacerbate the field defects caused by dermatochalasis and may explain the large inter-test fluctuation of field performance observed in all cases where visual field tests were repeated on more than one occasion without taping up the redundant upper lid skin (Fig. 3).

Due to the ability of dermatochalasis to cause field loss mimicking that of glaucoma the clinician must be sensitive to this possibility in order to avoid the commencement of unnecessary antiglaucomatous therapy. Patient 8 (Fig. 6) actually underwent a right trabeculectomy procedure in another unit before coming under our care. On retesting of his right field with the excess upper lid skin taped up no significant defect was found (Fig. 7).

In this series of ocular hypertensive patients it was possible to attribute blame for the field defects to dermatochalasis, since the optic discs were normal and the field defects were reversible on taping the lid. However, in patients with glaucoma the field loss could easily have been attributed to a deterioration of the glaucoma and the clinician must also be aware of factors capable of producing artefactual field loss in these cases.
In summary, we have shown that apparently mild dermatochalasis can be associated with quite marked defects in the central $24^{\circ}$ visual field when tested with automated static perimetry. The defects are most marked supero-temporally, but the scotomas can extend to involve the supero-nasal and inferotemporal field quadrants. The defects may show inter-test variability which may be due to the induction of mechanical ptosis exacerbated by fatigue. Finally, we conclude that dermatochalasis has potential to confound diagnostic automated visual field testing in glaucoma.

Presented as a poster at the Annual Congress of the Royal College of Ophthalmology, Edinburgh, 1996.

Key words: Dermatochalasis, Visual field loss, Glaucoma.

\section{REFERENCES}

1. Zeyen TG, Caprioli J. Progression of disc and field damage in early glaucoma. Arch Ophthalmol 1993; 111:62-5.

2. Katz J, Tielsch JM, Quigley H, Sommer A. Automated perimetry detects visual field loss before manual Goldmann perimetry. Ophthalmology 1995;102:21-6.

3. Webster AR, Luff AJ, Canning CR, Elkington AR. The effect of pilocarpine on the glaucomatous visual field. Br J Ophthalmol 1993;77:721-5.

4. Fisher RF. The influences of orbital contours and lid ptosis on the size of the peripheral visual field. Vision Res 1967;7:671-8.

5. Meyer DR, Stern JH, Jarvis JM. Evaluating the visual field effects of blepharoptosis using automated static perimetry. Ophthalmology 1993;100:651-9.

6. Cahill KV, Burns JA, Weber PA. The effect of blepharoptosis on the field of vision. Ophthalmic Plast Reconstr Surg 1987;3:121-5.

7. Meyer DR, Linberg JV, Powell SR, Odom JV. Quantitating the superior visual field loss associated with ptosis. Arch Ophthalmol 1989;107:840-3.

8. Patipa M. Visual field loss in primary gaze and reading gaze due to acquired blepharoptosis and visual field improvement following ptosis surgery. Arch Ophthalmol 1992;110:63-7.

9. Kanski JJ. Clinical ophthalmology, 2nd ed. London: Butterworth, 1989:14.

10. Committee for Ophthalmic Procedures Assessment. Functional indications for upper and lower eyelid blepharoplasty. Ophthalmology 1991;98:1461-3.

11. Hacker HD, Hollsten DA. Investigation of automated perimetry in the evaluation of patients for upper lid blepharoplasty. Ophthalmic Plastic Reconstr Surg 1992;8:250-5.

12. Beard C. Ptosis, 3rd ed. St Louis: CV Mosby, 1981:74.

13. Collin JR. A manual of systematic eyelid surgery, 2nd ed. Edinburgh: Churchill Livingstone, 1991:46. 